\title{
N-Acetylneuraminic Acid Supplementation Prevents High Fat Diet-Induced Insulin Resistance in Rats through Transcriptional and Nontranscriptional Mechanisms
}

\author{
Zhang Yida, ${ }^{1,2}$ Mustapha Umar Imam, ${ }^{1}$ Maznah Ismail, ${ }^{1,3}$ Norsharina Ismail, \\ Nur Hanisah Azmi, ${ }^{1}$ Waiteng Wong, ${ }^{1}$ Hadiza Altine Adamu, ${ }^{1}$ Nur Diyana Md Zamri, ${ }^{1}$ \\ Aini Ideris, ${ }^{4}$ and Maizaton Atmadini Abdullah ${ }^{5}$ \\ ${ }^{1}$ Laboratory of Molecular Biomedicine, Institute of Bioscience, Universiti Putra Malaysia, 43400 Serdang, Selangor, Malaysia \\ ${ }^{2}$ Cardiology Department, Affiliated Hospital of Chengde Medical University, Chengde, Hebei 067000, China \\ ${ }^{3}$ Department of Nutrition and Dietetics, Faculty of Medicine and Health Sciences, Universiti Putra Malaysia, 43400 Serdang, \\ Selangor, Malaysia \\ ${ }^{4}$ Faculty of Veterinary Medicine, Universiti Putra Malaysia, 43400 Serdang, Selangor, Malaysia \\ ${ }^{5}$ Department of Pathology, Faculty of Medicine and Health Sciences, Universiti Putra Malaysia, 43400 Serdang, \\ Selangor, Malaysia
}

Correspondence should be addressed to Mustapha Umar Imam; mustyimam@gmail.com and Maznah Ismail; maznahis@upm.edu.my

Received 25 June 2015; Revised 29 October 2015; Accepted 4 November 2015

Academic Editor: Sheba Mohankumar

Copyright (C) 2015 Zhang Yida et al. This is an open access article distributed under the Creative Commons Attribution License, which permits unrestricted use, distribution, and reproduction in any medium, provided the original work is properly cited.

\begin{abstract}
$\mathrm{N}$-Acetylneuraminic acid (Neu5Ac) is a biomarker of cardiometabolic diseases. In the present study, we tested the hypothesis that dietary Neu5Ac may improve cardiometabolic indices. A high fat diet (HFD) + Neu5Ac (50 or $400 \mathrm{mg} / \mathrm{kg}$ BW/day) was fed to rats and compared with HFD + simvastatin $(10 \mathrm{mg} / \mathrm{kg}$ BW/day) or HFD alone for 12 weeks. Weights and serum biochemicals (lipid profile, oral glucose tolerance test, leptin, adiponectin, and insulin) were measured, and mRNA levels of insulin signaling genes were determined. The results indicated that low and high doses of sialic acid (SA) improved metabolic indices, although only the oral glucose tolerance test, serum triglycerides, leptin, and adiponectin were significantly better than those in the HFD and HFD + simvastatin groups $(P<0.05)$. Furthermore, the results showed that only high-dose SA significantly affected the transcription of hepatic and adipose tissue insulin signaling genes. The data suggested that SA prevented HFD-induced insulin resistance in rats after 12 weeks of administration through nontranscriptionally mediated biochemical changes that may have differentially sialylated glycoprotein structures at a low dose. At higher doses, SA induced transcriptional regulation of insulin signaling genes. These effects suggest that low and high doses of SA may produce similar metabolic outcomes in relation to insulin sensitivity through multiple mechanisms. These findings are worth studying further.
\end{abstract}

\section{Introduction}

Sialic acids (SAs) are $\mathrm{N}$-acetylated derivatives of neuraminic acid that occur naturally in glycoproteins and gangliosides. SA is a biomarker of cardiometabolic diseases, where it is thought to be a consequence of long-term inflammation [1]. This claim is supported by the hypothesis that elevated SA levels in cardiovascular diseases may facilitate the resialylation of vascular endothelium in an attempt to reverse atherosclerosis [2]. Additionally, results of dietary supplementation with SA have not been consistent; some reports show that it promotes inflammation, hepatocellular cancer, and hemolytic-uremic syndrome $[3,4]$, while others have shown that it may be useful for brain development and for certain age-related disorders that cause reduced salivation $[5,6]$. What can be gleaned from the reports thus 
TABLE 1: Food composition and animal groups.

\begin{tabular}{|c|c|c|c|c|c|}
\hline Animal group & Normal pellet (\%) & Cholesterol/cholic acid (\%) & Palm oil (\%) & Starch $(\%)$ & Others \\
\hline Normal & 100 & & & & \\
\hline HFD & 65 & 5 & 20 & 10 & \\
\hline HFD + SIM & 65 & 5 & 20 & 10 & Simvastatin $(10 \mathrm{mg} / \mathrm{kg}$ BW/day) \\
\hline HFD + SAL & 65 & 5 & 20 & 10 & $50 \mathrm{mg} / \mathrm{kg}$ BW/day sialic acid \\
\hline $\mathrm{HFD}+\mathrm{SAH}$ & 65 & 5 & 20 & 10 & $400 \mathrm{mg} / \mathrm{kg}$ BW/day sialic acid \\
\hline
\end{tabular}

Normal pellet was acquired commercially while other diets were made in-house. HFD: high fat diet, SIM: simvastatin, SAL: low dose sialic acid, and SAH: high dose sialic acid.

far is that the nonhuman SA, N-glycolylneuraminic acid (Neu5Gc), not N-acetylneuraminic acid (Neu5Ac), is responsible for the deleterious effects of SA $[4,5]$.

There are numerous pharmacological and alternative therapies for cardiovascular diseases, but most of them have proven ineffective in curbing the rising burden of the diseases. This is driving the search for alternatives to currently available therapies [7]. Moreover, the rising burden of obesity and other risk factors for cardiometabolic diseases continually increase the prevalence of these diseases. If cardiometabolic diseases are to be effectively managed, there is a need for alternatives to the currently available therapies that control the risk factors and prevent the development of these diseases. To evaluate a potential alternative for the prevention of cardiometabolic diseases, we studied the effects of dietary supplementation with SA on the development of insulin resistance, which is a common denominator for cardiometabolic diseases [8]. Simvastatin is typically used to lower lipids, and because the animal model in the present study was given a diet rich in fats, it was used as a control. Additionally, we decided to use Neu5Ac since, which unlike Neu5Gc, it is the form that is widely reported to be beneficial. As a marker of cardiometabolic diseases, we hypothesized that dietary Neu5Ac could have far-reaching effects on cardiometabolic indices in view of the widespread incorporation of SA into multiple tissues in the body.

\section{Materials and Methods}

2.1. Materials. Neu5Ac was purchased from Carbosynth Limited (Compton, Berkshire, UK), while analytical grade ethanol was purchased from Merck (Darmstadt, Germany). Lipid profile kits were purchased from Randox Laboratories Ltd. (Crumlin, County Antrim, UK), while ELISA kits (leptin, insulin, and adiponectin) were purchased from Elabscience Biotechnology Co., Ltd. (Wuhan, China), and Millipore (Billerica, MA, USA), respectively. An RNA extraction kit was purchased from RBC Bioscience Corp. (Taipei, Taiwan), and a GenomeLab GeXP Start Kit was purchased from Beckman Coulter, Inc. (Miami, FL, USA). Simvastatin was purchased from Pfizer (New York, NY, USA), and RCL2 solution was purchased from Alphelys (Toulouse, France). Cholesterol and cholic acid were purchased from Amresco (Solon, OH, USA) and Santa Cruz Biotechnology (Santa Cruz, CA, USA), respectively. Standard rat pellet was purchased from Specialty Feeds (Glen Forrest, WA, USA), while palm oil was supplied by Yee Lee Edible Oils Sdn. Bhd. (Perak, Malaysia).

2.2. Animal Study. The Animal Care and Use Committee (ACUC) of the Faculty of Medicine and Health Sciences, Universiti Putra Malaysia, approved the use of animals in this study (project approval number UPM/IACUC/AUPR011/2014), and the animals were handled as stipulated by the guidelines for the use of animals. Sprague Dawley rats (10-week-old, 230-280 g, $n=30$ ) were housed in individual cages at $25 \pm 2^{\circ} \mathrm{C}$, with $12 / 12 \mathrm{~h}$ light/dark cycle, and allowed to acclimatize for 2 weeks with free access to normal pellet and water. The rats were then assigned to one of five groups ( $n=6$, Table 1): normal group fed with standard rat pellet (335 Kcal/100 g), high fat diet (HFD) group fed a HFD alone $(448 \mathrm{Kcal} / 100 \mathrm{~g})$, simvastatin group fed with HFD + oral gavage of $10 \mathrm{mg} / \mathrm{kg}$ BW simvastatin/day (HFD + SIM), and $\mathrm{SA}$ groups that received HFD + daily oral gavage of 50 or $400 \mathrm{mg} / \mathrm{kg}$ BW SA (HFD + SAL and HFD + SAH, resp.). Simvastatin was chosen because it is the standard drug used to manage hyperlipidemia and associated metabolic perturbations [9], similar to what HFD induces in rats. Diets were formulated in-house except for the normal pellet and were given to the rats for 12 weeks, after which they were euthanized and their blood and organs (liver and visceral adipose tissues) were collected for further studies. Tissue samples were immediately washed with normal saline and preserved in chilled RCL-2 solution, which was then transferred to $-80^{\circ} \mathrm{C}$ until RNA was extracted. During the intervention, food intake was calculated daily by subtracting the leftover food from what was added the previous day, while body weight was recorded.

2.3. Serum Adiponectin, Leptin, and Insulin. Serum from blood collected in plain tubes was used for measurements of adiponectin, leptin, and insulin using the respective ELISA kits according to the manufacturer's instructions. Absorbances were read on a BioTeK Synergy H1 Hybrid Reader (BioTek Instruments, Inc., Winooski, VT, USA) at the appropriate wavelengths $(450 \mathrm{~nm}$ for insulin and leptin, and $450 \mathrm{~nm}$ and $590 \mathrm{~nm}$ for adiponectin). The results were analyzed on http://www.myassays.com/ using four parametric test curves: adiponectin $\left(R^{2}=0.9914\right)$, insulin $\left(R^{2}=1\right)$, and leptin $\left(R^{2}=0.9996\right)$.

2.4. Biochemical Analyses. Lipid profile analyses were performed using serum from blood collected at the end of the study by cardiac puncture after an overnight fast. Samples 
were analyzed using Randox analytical kits according to the manufacturer's instructions with a Selectra XL instrument (Vita Scientific, Dieren, Netherlands). After an overnight fast, the oral glucose tolerance test (OGTT) was performed by oral gavage of $2 \mathrm{~g} / \mathrm{kg} \mathrm{BW} \mathrm{D}$-glucose to every rat, and the blood glucose levels were measured at $0,30,60$, and 120 minutes via tail vein puncture, using a glucometer (Roche Diagnostics, Indianapolis, IN, USA). The area under the curve for glucose in the OGTT was calculated as reported previously [10]. Then, homeostatic model assessment of insulin resistance (HOMAIR), a measure of insulin sensitivity, was computed from the fasting plasma glucose and insulin levels using the formula HOMA-IR $=$ (fasting glucose level $(\mathrm{mg} / \mathrm{dL}) /$ fasting plasma insulin $(\mu \mathrm{U} / \mathrm{mL})) / 2430$ [11].

2.5. Gene Expression Study. Primers for the gene expression study were designed using the Rattus norvegicus gene sequences from the National Center for Biotechnology Information website (http://www.ncbi.nlm.nih.gov/nucleotide/) and tagged with an 18-nucleotide universal forward and 19nucleotide universal reverse sequence, respectively. Primers (Table 2) were supplied by Integrated DNA Technologies (Singapore) and reconstituted in RNAse free water. Extracted RNA (20 ng) from liver and adipose tissues was used for reverse transcription and PCR according to the GenomeLab GeXP Start Kit protocol (Beckman Coulter, USA) using the conditions shown in Table 2 . PCR products $(1 \mu \mathrm{L})$ were analyzed on a GeXP genomelab genetic analysis system (Beckman Coulter, Inc., Miami, FL, USA) after mixing with sample loading solution and DNA size standard 400 as recommended by the manufacturer. Results were analyzed with the Fragment Analysis module of the GeXP system software and normalized on the eXpress Profiler software. Fold changes were calculated by dividing the expression value for the different treatment groups by the expression value for the normal group.

2.6. Data Analysis. The means \pm standard deviation $(n=6)$ of the groups was used for the analyses. One-way analysis of variance (ANOVA) with Tukey's post hoc test was performed using SPSS 17.0 software (SPSS, Inc., Chicago, IL, USA) to assess the level of significance of the differences between means with a cutoff of $P<0.05$.

\section{Results}

3.1. Body Weight Changes. Food intake was similar for all the groups throughout the intervention period (Table 3), but differences in weight gain were observed between the HFD and other groups at the end of 12 weeks (Figure 1); the highest weight gain between the beginning and end of the study (50\%) was in the HFD group, followed by the normal group (47\%), while the HFD + SIM group had the lowest weight gain (40\%). The HFD + SAH and HFD + SAL groups had weight gains of $42 \%$ and $46 \%$, respectively.

3.2. OGTT, Insulin, HOMA-IR, and Lipid Profile. The OGTT, insulin, HOMA-IR, and lipid profile results are shown in

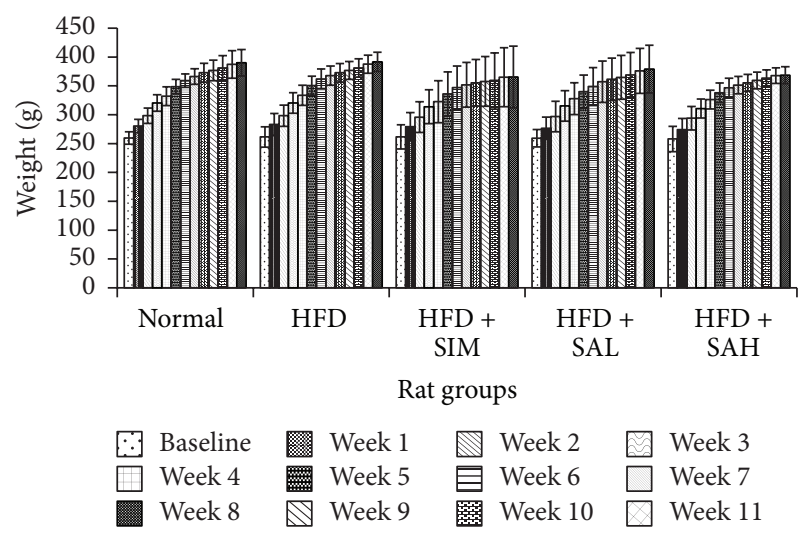

(a)

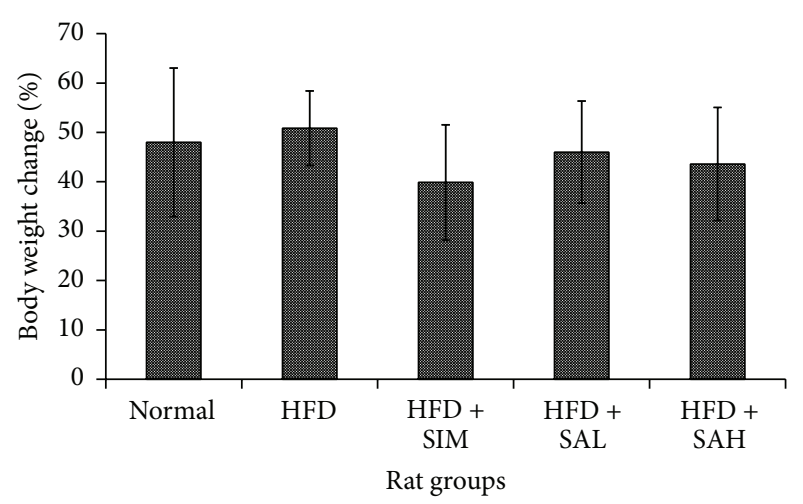

(b)

FIGURE 1: Effects of SA on body weight changes in HFD-fed rats over 12 weeks. (a) Total body weight changes. (b) Percentage of body weight changes. Groups are the same as Table 1. No significant differences were observed between the groups' actual weights, but total weight gain was the highest in the HFD and normal groups ( $50 \%$ and $47 \%$, resp.), while the HFD + simvastatin group had the lowest ( $40 \%$ increase), followed by the HFD + SAH and HFD + SAL groups ( $42 \%$ and $46 \%$, resp.).

Table 3 and Figure 2. The results indicated that SA improved lipid profile values and insulin sensitivity (HOMA-IR) of rats, although only the triglycerides were significantly different $(P<0.05)$ in comparison with the HFD group (Table 3$)$. Moreover, OGTT results showed that the HFD + SAH and HFD + SAL groups, unlike the HFD and HFD + SIM groups, had better insulin sensitivity (Figure 2); the HFD + SAH and HFD + SAL groups had better glycemic response on OGTT and lower area under the curve for glucose over $120 \mathrm{~min}$ of OGTT $(P<0.05)$.

3.3. Serum Adiponectin and Leptin. Table 3 shows the serum adiponectin and leptin results. From the table, elevated leptin and reduced adiponectin levels were observed. The leptin level was significantly lower in the HFD + SIM, HFD + SAH, and HFD + SAL groups compared to the HFD group $(P<$ 0.05). Adiponectin level in the HFD + SIM group was also lower than that of the HFD group, while the HFD + SAH and HFD + SAL groups had higher levels, despite being not significantly different from the HFD group. 


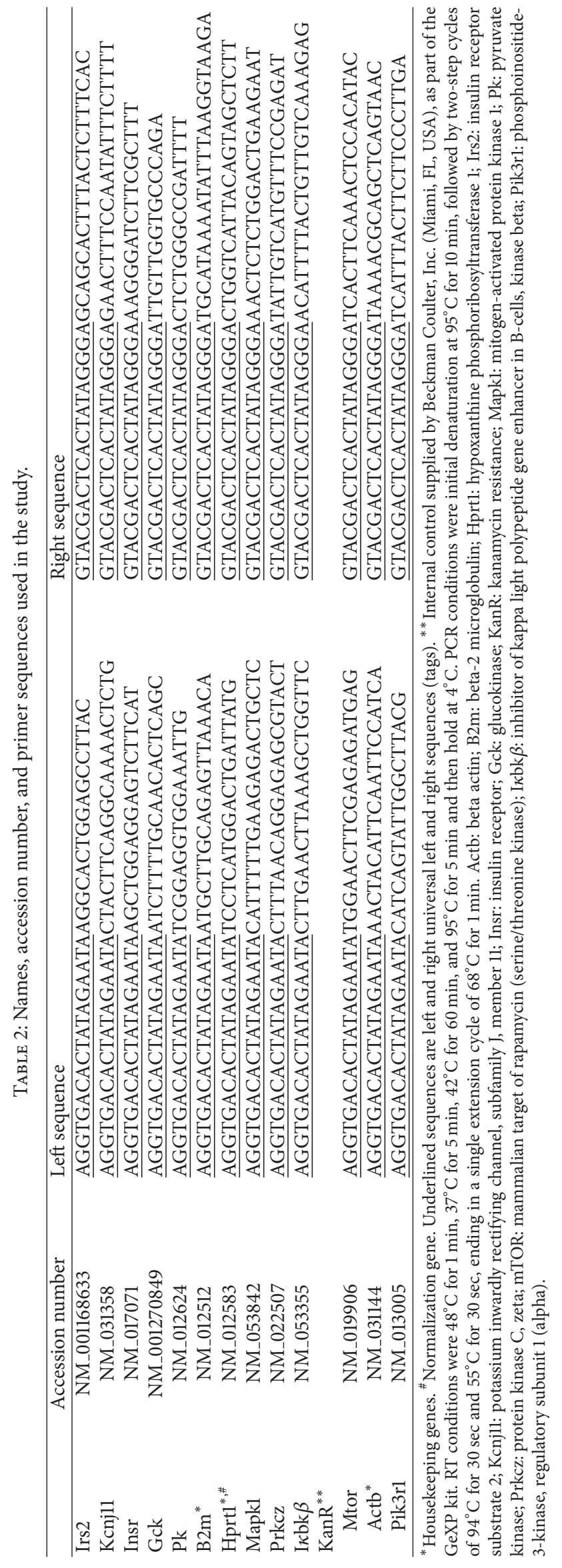




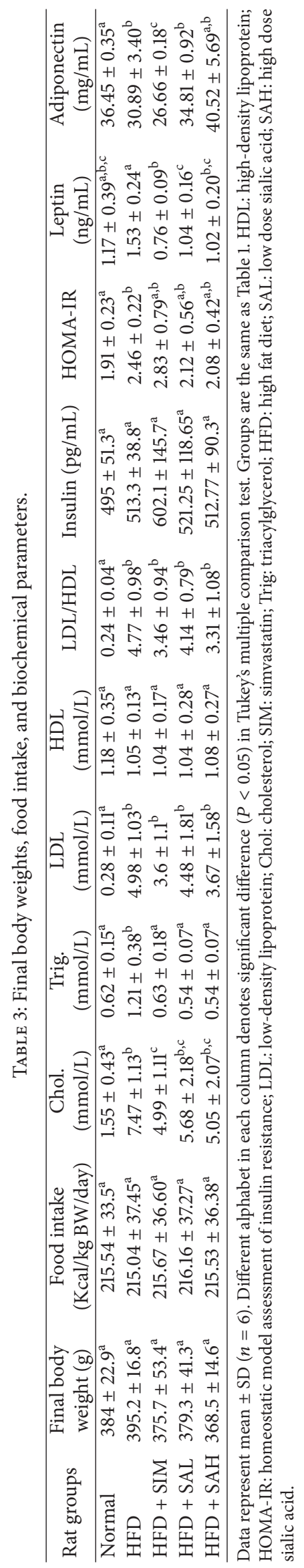




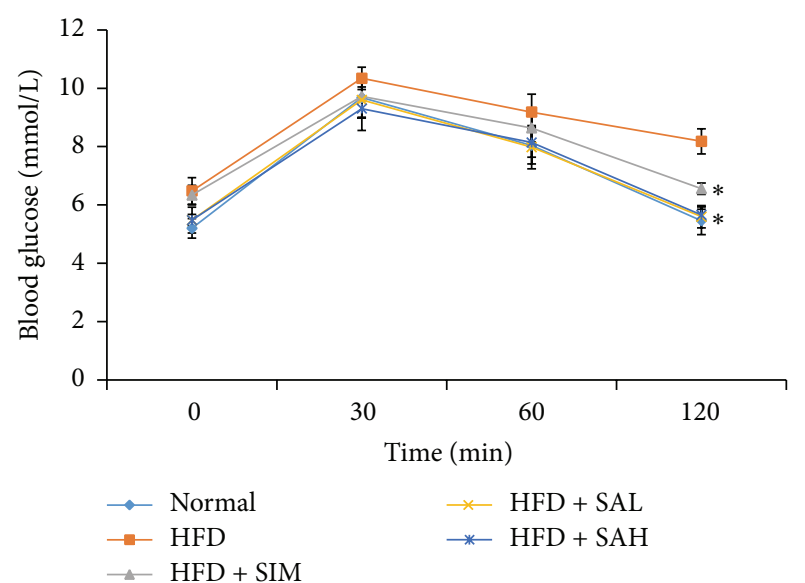

(a)

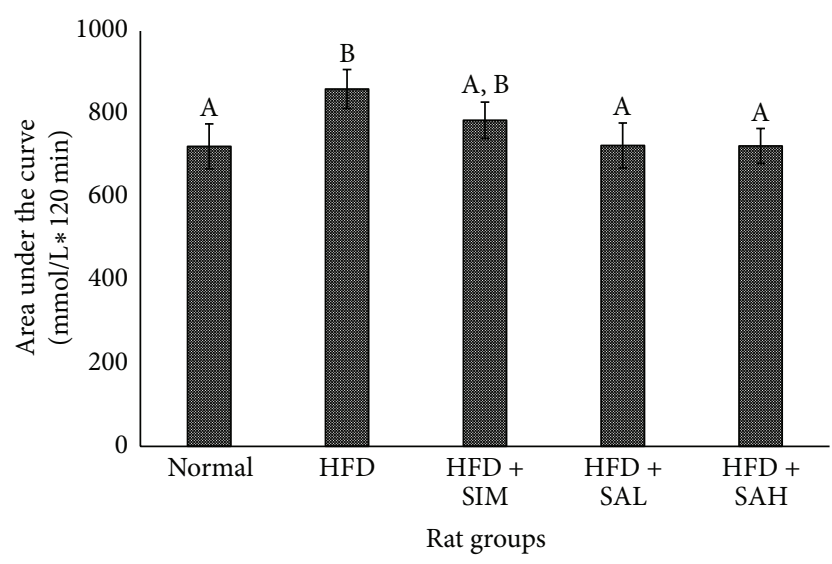

(b)

FIGURE 2: Effects of SA on oral glucose tolerance test in HFD-fed rats. (a) Glycemic response to $2 \mathrm{~g}$ glucose $/ \mathrm{kg}$ BW over $120 \mathrm{~min}$. (b) Area under the curve for glucose after $120 \mathrm{~min}$. Groups are the same as Table $1{ }^{*}$ indicates statistically significant difference $(P<0.05)$ in comparison with HFD. Different letters on bars in (b) indicate a statistically significant difference $(P<0.05)$.

\subsection{Hepatic and Adipose Tissue mRNA Levels of Insulin} Signaling Genes. Figures 3 and 4 show the effects of SA on hepatic and adipose tissue mRNA levels of insulin signaling genes, respectively. The HFD + SAH group had upregulation of the glucokinase (Gck), potassium inwardly rectifying channel, subfamily J, member 11 (KCNJ11), mammalian target of rapamycin (mTOR), phosphoinositide-3-kinase (Pi3k), and prkcz-protein kinase C, zeta (Prckz) genes in both liver and adipose tissues. In addition, it had downregulation of the inhibitor of kappa light polypeptide gene enhancer in B-cells, kinase beta $(\mathrm{I} \kappa \mathrm{bk} \beta)$, and mitogen-activated protein kinase (Mapk1) in the liver, while it upregulated pyruvate kinase $(\mathrm{Pk})$ in the adipose tissue. Other genes involved in the insulin signaling pathway were not changed by SA (Figures 3 and 4).

\section{Discussion}

As can be recalled, the HFD + SIM, HFD + SAH, and HFD + SAL groups had lower weight gains in comparison with the HFD group. Simvastatin has weight-reducing properties [12] and hence the lower weight gain observed. SA, on the other hand, has not been reported to reduce weight, and the observations from this study suggest that it may regulate weight gain. Therefore, we hypothesized that this effect may have been due to differential sialylation or resialylation of glycoprotein or glycolipids with implications on weight gain. Moreover, Zinc $\alpha 2$-glycoprotein (ZAG), a glycoprotein, is an established marker for fat catabolism [13], and dietary SA may have affected its sialylation or that of similar yet unknown glycoprotein adipokines, with resultant changes in their functions due to changes in sialylation status. This is an area worth evaluating further, especially since the metabolism of such glycoproteins may in itself be influenced by the degree of sialylation $[14,15]$. Additionally, the changes in lipid profiles and insulin sensitivity markers in the HFD + SAH and HFD + SAL groups may also have been due to differential sialylation of glycoproteins like ZAG, with resultant modulation of various metabolic pathways. The lower weight gains in the HFD + SAH and HFD + SAL groups may also have contributed to the metabolic outcomes observed in these groups, since lower weights have been associated with lower lipid profiles and improved insulin sensitivity [8]. Elevated leptin and reduced adiponectin levels are associated with cardiometabolic diseases [16], and in the present study, a similar pattern was observed for the HFD group (Table 3 ). Conversely, SA reduced leptin level significantly lower than that in the HFD group and increased the adiponectin level, albeit not significantly. Simvastatin, however, reduced both the leptin and adiponectin levels. The effects of SA on adiponectin and leptin indicated that it could improve cardiometabolic indices, since these markers are predictors of metabolic diseases [16].

The gene expression data from this study showed that the HFD + SIM and HFD groups had transcriptional changes of insulin signaling genes that tended towards insulin resistance. Furthermore, the data showed that the HFD + SAH group attenuated the HFD-induced transcriptional changes, which tended towards improved insulin signaling. Specifically, it upregulated the expression of a central mediator of the intracellular signal transduction of insulin sensing ( $\mathrm{Pi} 3 \mathrm{k})$, whose transcriptional downregulation has been linked with obesityinduced insulin resistance $[17,18]$. Similarly, the upregulation of mTOR [19] and prkcz [20] and downregulation of Mapk1 [21] and $\operatorname{I} \kappa \mathrm{bk} \beta$ [22] especially in the HFD + SAH group suggested improved insulin sensitivity, which may have been mediated through increased dephosphorylation of insulin receptor substrate (IRS) with consequent increase in IRSmediated insulin action via activation of Pi3k [17, 23]. The data in this study support this hypothesis, since the expression of Pi3k was increased but not that of IRS. Also, in the present study, SA upregulated Gck (liver and adipose tissues) and $\mathrm{Pk}$ (adipose tissue), which are associated with enhanced glucose sensing and homeostasis and elevated cellular adenosine triphosphate (ATP) levels [24]. Elevated cellular ATP will consequently increase KCNJ11, which is reported to regulate ion channels involved in glucose sensing [25].

Taken together, the data showed that although simvastatin improved lipid profiles, it increased insulin resistance, 


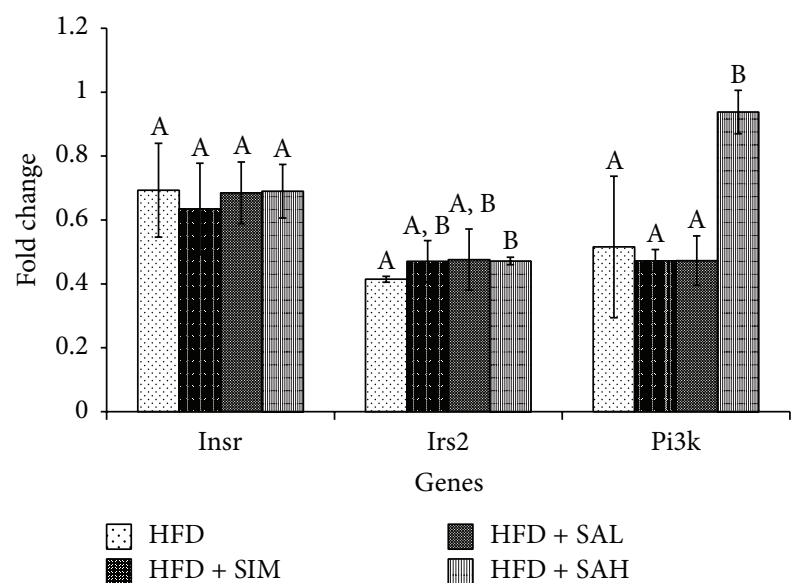

(a)

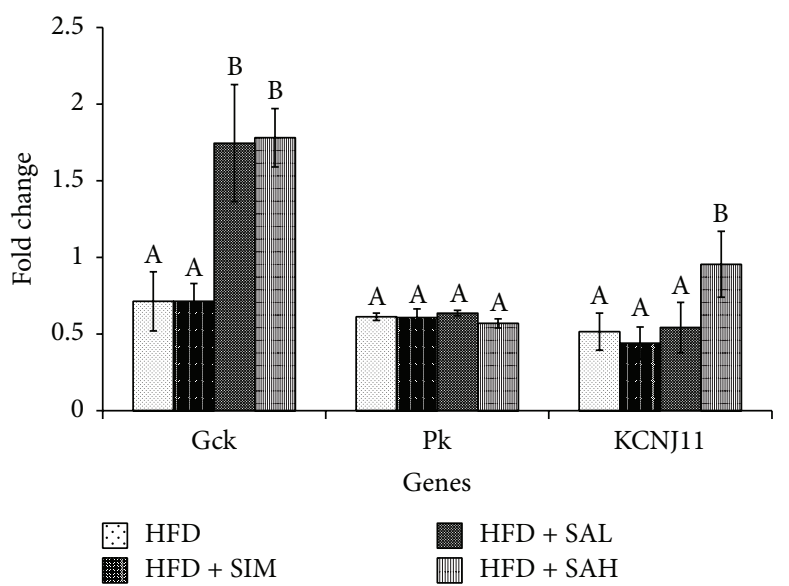

(b)

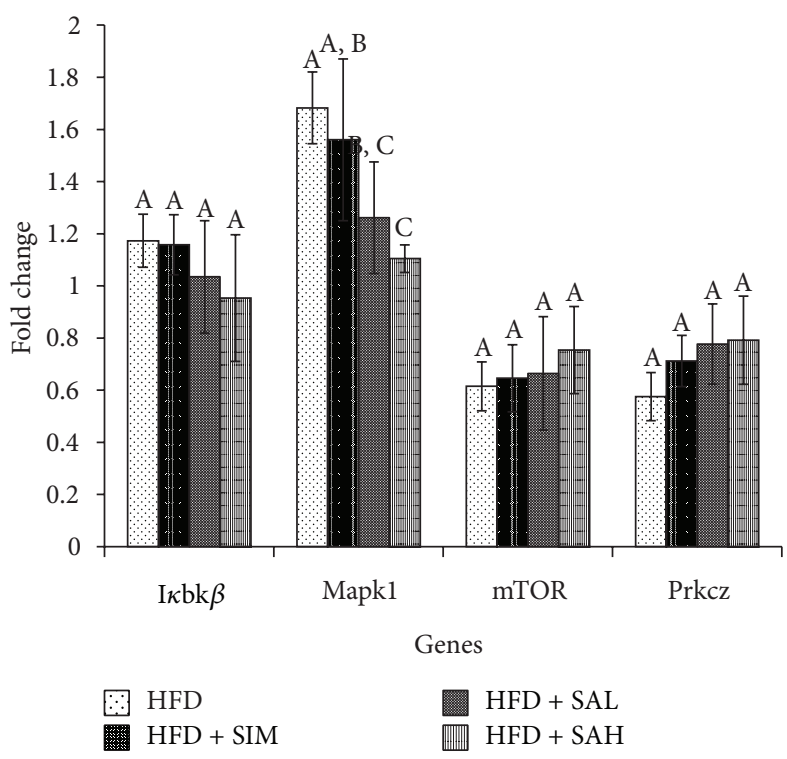

(c)

FIGURE 3: Effects of SA on hepatic mRNA levels of (a) insulin receptor (Insr), insulin receptor substrate (Irs) 2, and phosphoinositide-3-kinase (PI3K), (b) glucokinase (Gck), potassium inwardly rectifying channel, subfamily J, member 11 (KCNJ11), and pyruvate kinase-liver isoform (L-Pk), and (c) mammalian target of rapamycin (mTOR), protein kinase C, zeta (Prkcz), inhibitor of kappa light polypeptide gene enhancer in B-cells, kinase beta $(\mathrm{I} \kappa \mathrm{BK} \beta)$, and mitogen-activated protein kinase (MAPK) 1 in HFD-fed rats. Groups are the same as Table 1 . Different letters on bars representing each group indicate a statistically significant difference $(P<0.05)$.

as reported previously [26]. SA, on the other hand, improved lipid profiles and prevented HFD-induced insulin resistance. Serum insulin levels in the HFD + SIM, HFD + SAH, and HFD + SAL groups were similar, but insulin sensitivity was better in the HFD + SAH and HFD + SAL groups, suggesting that the effects of SA may have been mediated at the insulin signaling level. The effects of SA on mRNA levels of hepatic and adipose tissue insulin signaling genes confirmed our hypothesis. However, despite improved insulin sensitivity in the HFD + SAH and HFD + SAL groups, only the $\mathrm{HFD}+\mathrm{SAH}$ group showed upregulation of the insulin signaling genes, suggesting that the effects of SA may be both transcriptional (at higher doses) and nontranscriptional (at lower and higher doses). Earlier, we hypothesized that the improvements in weight and other metabolic indices observed due to SA could have been due to its effects on glycoprotein sialylation. Moreover, glycoprotein sialylation is reportedly influenced by the degree of metabolic flux [14, 15], and dietary SA administration could have influenced the flux towards increased sialylation of glycoprotein structures that influenced cardiometabolic indices. These effects induced by SA are likely to be transient, since reduced SA flux could rapidly reverse any changes produced. However, transcriptionally mediated changes at higher doses of SA may produce longer-lasting effects $[26,27]$. Therefore, from the findings in this study, we propose that SA may be able to 


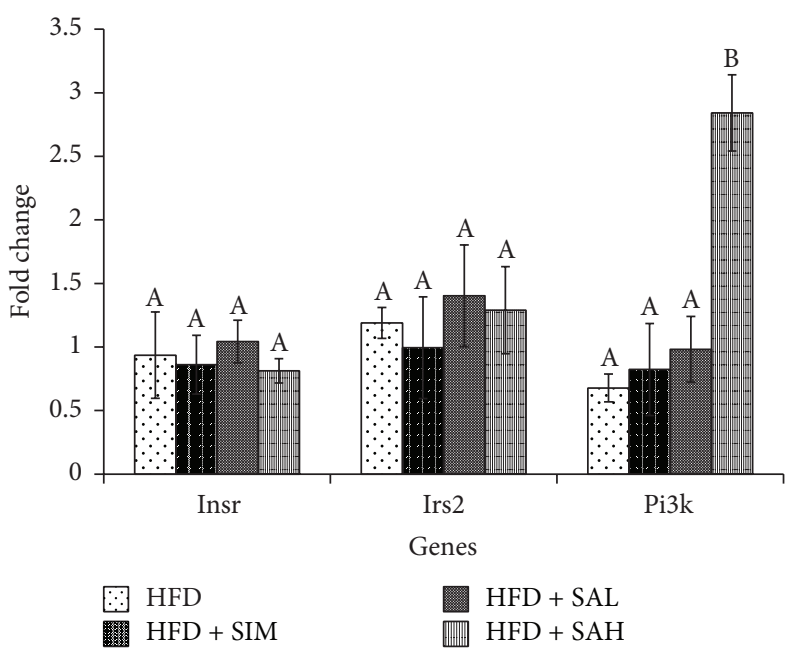

(a)

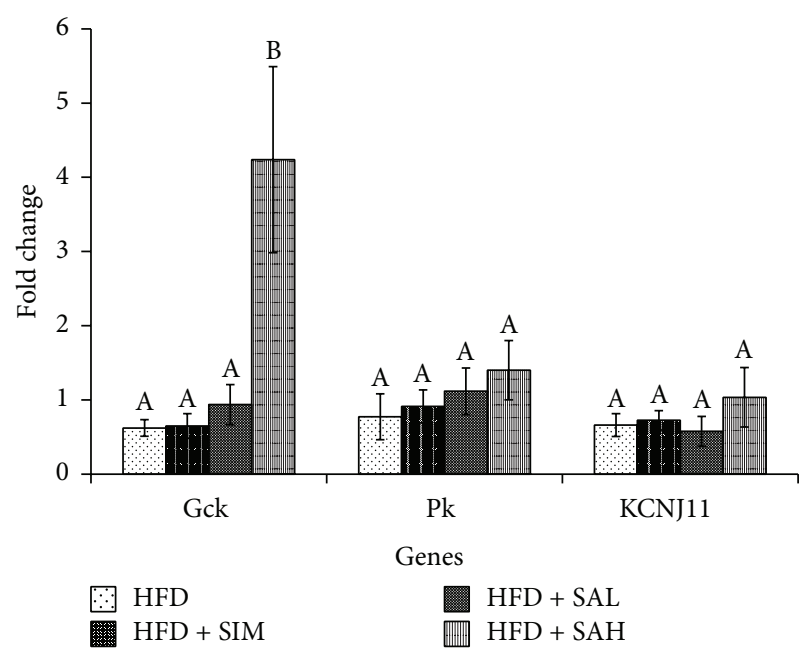

(b)

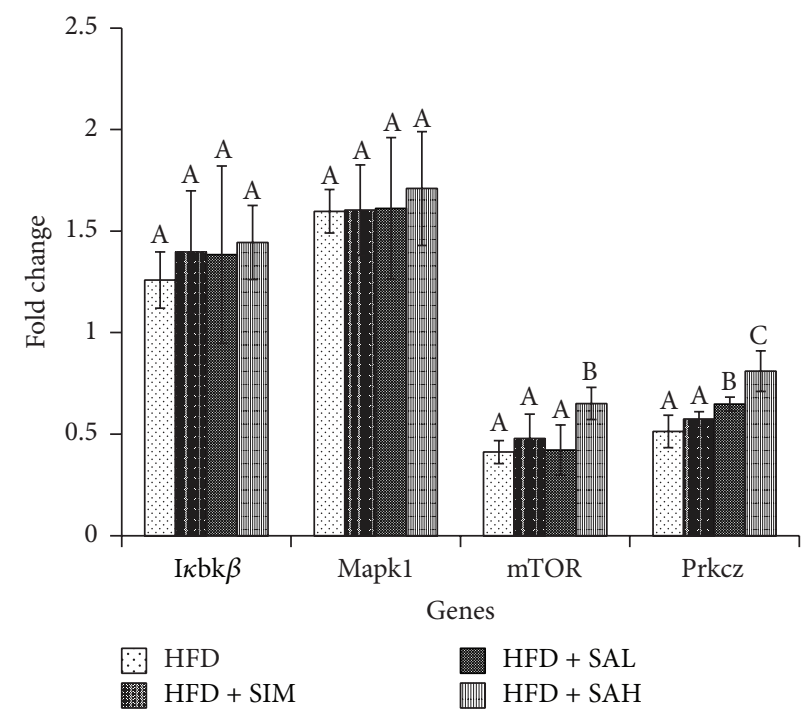

(c)

FIGURE 4: Effects of SA on adipose tissue mRNA levels of (a) insulin receptor (Insr), insulin receptor substrate (Irs) 2, and phosphoinositide3-kinase (PI3K), (b) glucokinase (Gck), potassium inwardly rectifying channel, subfamily J, member 11 (KCNJ11), and pyruvate kinase-liver isoform (L-Pk), and (c) mammalian target of rapamycin (mTOR), protein kinase C, zeta (Prkcz), inhibitor of kappa light polypeptide gene enhancer in B-cells, kinase beta $(\mathrm{I} \kappa \mathrm{BK} \beta)$, and mitogen-activated protein kinase (MAPK) 1 in HFD-fed rats. Groups are the same as Table 1. Different letters on bars representing each group indicate a statistically significant difference $(P<0.05)$.

prevent insulin resistance through transcriptional regulation of insulin signaling genes (Figure 5) and nontranscriptional mechanisms, depending on the concentration used.

\section{Conclusions}

We demonstrated that SA prevents HFD-induced insulin resistance through transcriptional and nontranscriptional mechanisms. At lower doses, sialylation of glycoprotein targets may be responsible for the preventive effects of SA against insulin resistance. In addition, at higher doses, transcriptional regulation of insulin signaling genes may provide longer-lasting effects. These findings are worth evaluating further.

\section{Abbreviations}

Gck: Glucokinase

HFD: $\quad$ High fat diet

HOMA-IR: Homeostatic model assessment of insulin resistance

$\mathrm{I} \kappa \mathrm{bk} \beta$ : $\quad$ Inhibitor of kappa light polypeptide gene enhancer in B-cells, kinase beta

IRS: Insulin receptor substrate

KCNJ11: Potassium inwardly rectifying channel, subfamily J, member 11

Mapk: Mitogen-activated protein kinase

mTOR: Mammalian target of rapamycin

OGTT: Oral glucose tolerance test

Pi3k: Phosphoinositide-3-kinase 


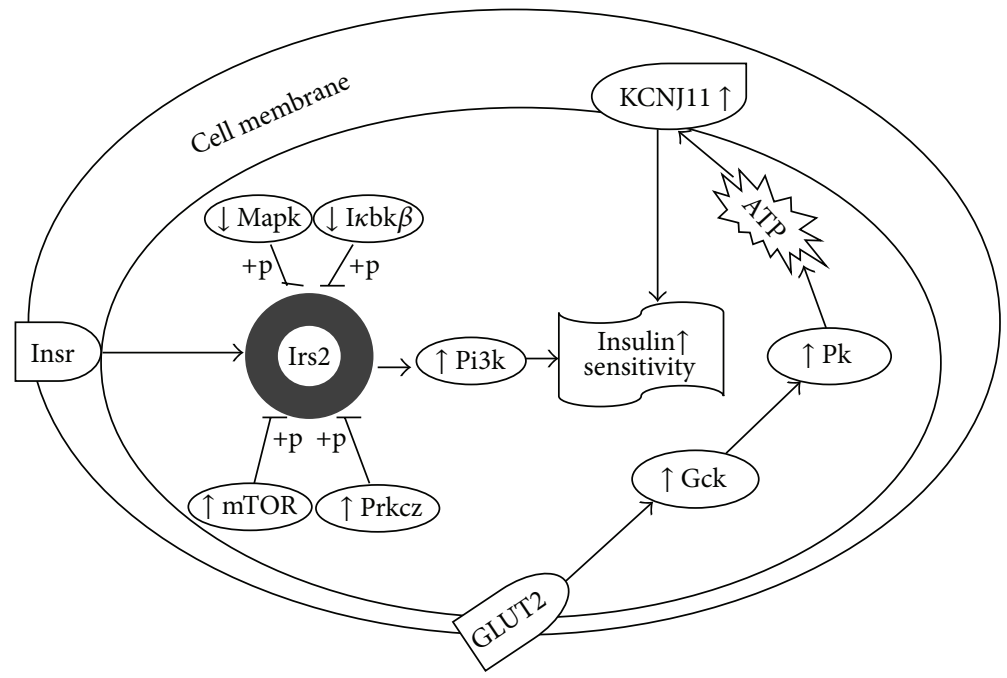

FIGURE 5: Proposed schematic showing targets of SA transcriptional regulation of insulin signaling pathway. SA prevents insulin resistance in HFD-fed rats by influencing the transcriptional regulation of multiple genes. Gck: glucokinase; I $k b k \beta$ : inhibitor of kappa light polypeptide gene enhancer in B-cells, kinase beta; IRS: insulin receptor substrate; KCNJ11: potassium inwardly rectifying channel, subfamily J, member 11; Mapk: mitogen-activated protein kinase; mTOR: mammalian target of rapamycin; Pi3k: phosphoinositide-3-kinase; Pk: pyruvate kinase; and Prkcz: protein kinase C, zeta.

Pk: Pyruvate kinase

Prkcz: Protein kinase C, zeta

SA: $\quad$ Sialic acid

SAH: High-dose sialic acid

SAL: Low dose sialic acid.

\section{Conflict of Interests}

The authors declare that there is no conflict of interests regarding the publication of this paper.

\section{Authors' Contribution}

Zhang Yida and Maznah Ismail participated in conception of idea and research design. Zhang Yida, Mustapha Umar Imam, Norsharina Ismail, Nur Hanisah Azmi, Waiteng Wong, Hadiza Altine Adamu, and Nur Diyana Md Zamri participated in conduct of research and experimentation. Zhang Yida and Mustapha Umar Imam participated in data analyses. Zhang Yida and Mustapha Umar Imam helped in drafting of the paper. Mustapha Umar Imam, Maznah Ismail, Aini Ideris, and Maizaton Atmadini Abdullah helped in review and approval of the final paper.

\section{Acknowledgments}

The authors thank the Ministry of Science, Technology, and Innovation (MOSTI), Malaysia, for sponsoring this research (e-Sciencefund 02-01-04-SF1453), and the staff of the Laboratory of Molecular Biomedicine, for their assistance during the study.

\section{References}

[1] K. P. Gopaul and M. A. Crook, "Sialic acid: a novel marker of cardiovascular disease?" Clinical Biochemistry, vol. 39, no. 7, pp. 667-681, 2006.

[2] G. Lindberg, "Resialylation of sialic acid deficit vascular endothelium, circulating cells and macromolecules may counteract the development of atherosclerosis: a hypothesis," Atherosclerosis, vol. 192, no. 2, pp. 243-245, 2007.

[3] A. N. Samraj, H. Laubli, O. Pearce et al., "A diet-derived sialic acid promotes inflammation and hepatocellular cancer," Cancer Research, vol. 74, Article ID LB-156, 2014.

[4] J. C. Löfling, A. W. Paton, N. M. Varki, J. C. Paton, and A. Varki, "A dietary non-human sialic acid may facilitate hemolyticuremic syndrome," Kidney International, vol. 76, no. 2, pp. 140144, 2009.

[5] B. Wang, "Sialic acid is an essential nutrient for brain development and cognition," Annual Review of Nutrition, vol. 29, pp. 177-222, 2009.

[6] N. Sprenger, M. Julita, D. Donnicola, and A. Jann, "Sialic acid feeding aged rats rejuvenates stimulated salivation and colon enteric neuron chemotypes," Glycobiology, vol. 19, no. 12, pp. 1492-1502, 2009.

[7] J. M. Hollander and J. I. Mechanick, "Complementary and alternative medicine and the management of the metabolic syndrome," Journal of the American Dietetic Association, vol. 108, no. 3, pp. 495-509, 2008.

[8] R. A. DeFronzo and E. Ferrannini, "Insulin resistance: a multifaceted syndrome responsible for NIDDM, obesity, hypertension, dyslipidemia, and atherosclerotic cardiovascular disease," Diabetes Care, vol. 14, no. 3, pp. 173-194, 1991.

[9] A. S. Antonopoulos, M. Margaritis, R. Lee, K. Channon, and C. Antoniades, "Statins as anti-inflammatory agents in atherogenesis: molecular mechanisms and lessons from the recent 
clinical trials," Current Pharmaceutical Design, vol. 18, no. 11, pp. 1519-1530, 2012.

[10] M. M. Tai, "A mathematical model for the determination of total area under glucose tolerance and other metabolic curves," Diabetes Care, vol. 17, no. 2, pp. 152-154, 1994.

[11] J. Cacho, J. Sevillano, J. de Castro, E. Herrera, and M. P. Ramos, "Validation of simple indexes to assess insulin sensitivity during pregnancy in Wistar and Sprague-Dawley rats," The American Journal of Physiology_Endocrinology and Metabolism, vol. 295, no. 5, pp. E1269-E1276, 2008.

[12] A. A. Adeneye, O. O. Adeyemi, and E. O. Agbaje, "Anti-obesity and antihyperlipidaemic effect of Hunteria umbellata seed extract in experimental hyperlipidaemia," Journal of Ethnopharmacology, vol. 130, no. 2, pp. 307-314, 2010.

[13] C. Bing, T. Mracek, D. Gao, and P. Trayhurn, "Zinc- $\alpha 2-$ glycoprotein: an adipokine modulator of body fat mass," International Journal of Obesity, vol. 34, no. 11, pp. 1559-1565, 2010.

[14] R. T. Almaraz, Y. Tian, R. Bhattarcharya et al., "Metabolic flux increases glycoprotein sialylation: implications for cell adhesion and cancer metastasis," Molecular \& Cellular Proteomics, vol. 11, no. 7, 2012.

[15] R. K. Margolis, R. U. Margolis, C. Preti, and D. Lai, "Distribution and metabolism of glycoproteins and glycosaminoglycans in subcellular fractions of brain," Biochemistry, vol. 14, no. 22, pp. 4797-4804, 1975.

[16] A. Yadav, M. A. Kataria, V. Saini, and A. Yadav, "Role of leptin and adiponectin in insulin resistance," Clinica Chimica Acta, vol. 417, pp. 80-84, 2013.

[17] E. Hirsch, C. Costa, and E. Ciraolo, "Phosphoinositide 3-kinases as a common platform for multi-hormone signaling," Journal of Endocrinology, vol. 194, no. 2, pp. 243-256, 2007.

[18] S. E. Kahn, R. L. Hull, and K. M. Utzschneider, "Mechanisms linking obesity to insulin resistance and type 2 diabetes," Nature, vol. 444, no. 7121, pp. 840-846, 2006.

[19] M. Laplante and D. M. Sabatini, "mTOR signaling at a glance," Journal of Cell Science, vol. 122, no. 20, pp. 3589-3594, 2009.

[20] G. Bandyopadhyay, M. P. Sajan, Y. Kanoh et al., "PKC$\xi$ mediates insulin effects on glucose transport in cultured preadipocyte-derived human adipocytes," Journal of Clinical Endocrinology and Metabolism, vol. 87, no. 2, pp. 716-723, 2002.

[21] M. Igarashi, H. Wakasaki, N. Takahara et al., "Glucose or diabetes activates p38 mitogen-activated protein kinase via different pathways," The Journal of Clinical Investigation, vol. 103, no. 2, pp. 185-195, 1999.

[22] M. C. Arkan, A. L. Hevener, F. R. Greten et al., "IKK- $\beta$ links inflammation to obesity-induced insulin resistance," Nature Medicine, vol. 11, no. 2, pp. 191-198, 2005.

[23] M. J. Brady, "IRS2 takes center stage in the development of type 2 diabetes," The Journal of Clinical Investigation, vol. 114, no. 7, pp. 886-888, 2004.

[24] S. Lenzen, "A fresh view of glycolysis and glucokinase regulation: history and current status," Journal of Biological Chemistry, vol. 289, no. 18, pp. 12189-12194, 2014.

[25] P. Proks, C. Girard, and F. M. Ashcroft, "Functional effects of KCNJ11 mutations causing neonatal diabetes: enhanced activation by MgATP," Human Molecular Genetics, vol. 14, no. 18, pp. 2717-2726, 2005.

[26] B. Desvergne, L. Michalik, and W. Wahli, "Transcriptional regulation of metabolism," Physiological Reviews, vol. 86, no. 2, pp. 465-514, 2006.
[27] P. Hammonds, P. N. Schofield, S. J. H. Ashcroft, R. Sutton, and D. W. R. Gray, "Regulation and specificity of glucose-stimulated insulin gene expression in human islets of Langerhans," FEBS Letters, vol. 223, no. 1, pp. 131-137, 1987. 


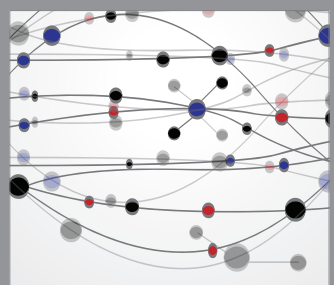

The Scientific World Journal
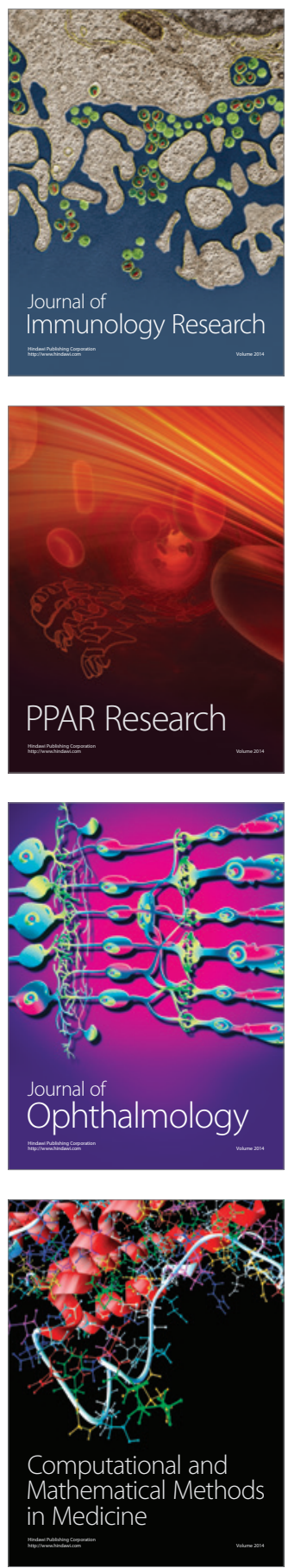

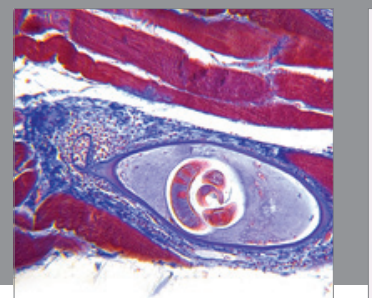

Gastroenterology

Research and Practice
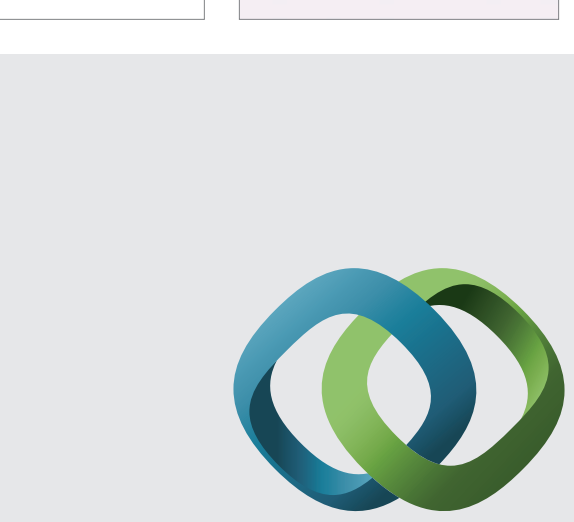

\section{Hindawi}

Submit your manuscripts at

http://www.hindawi.com
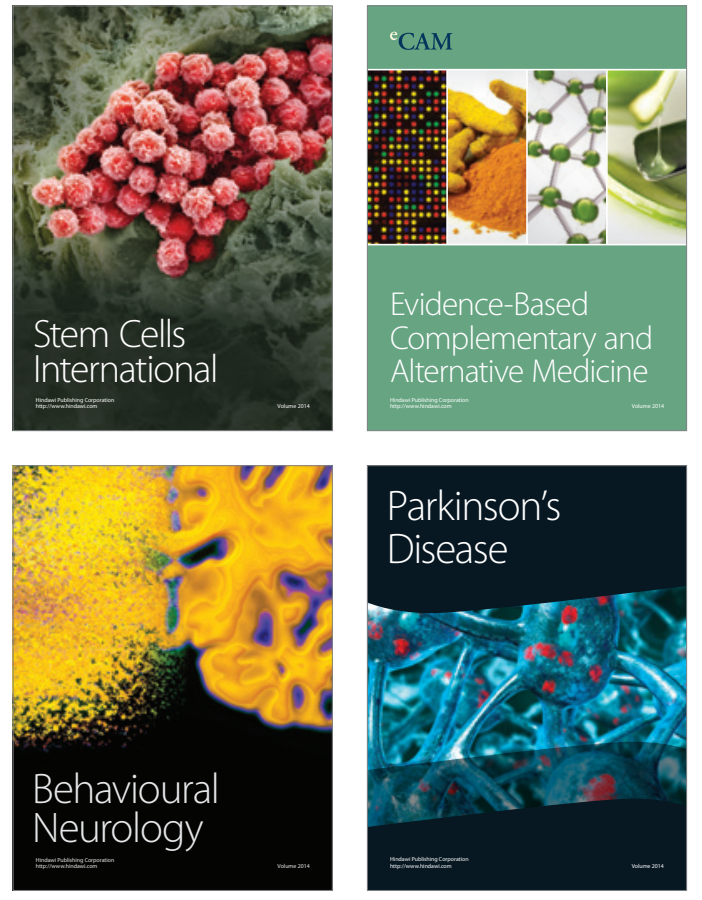
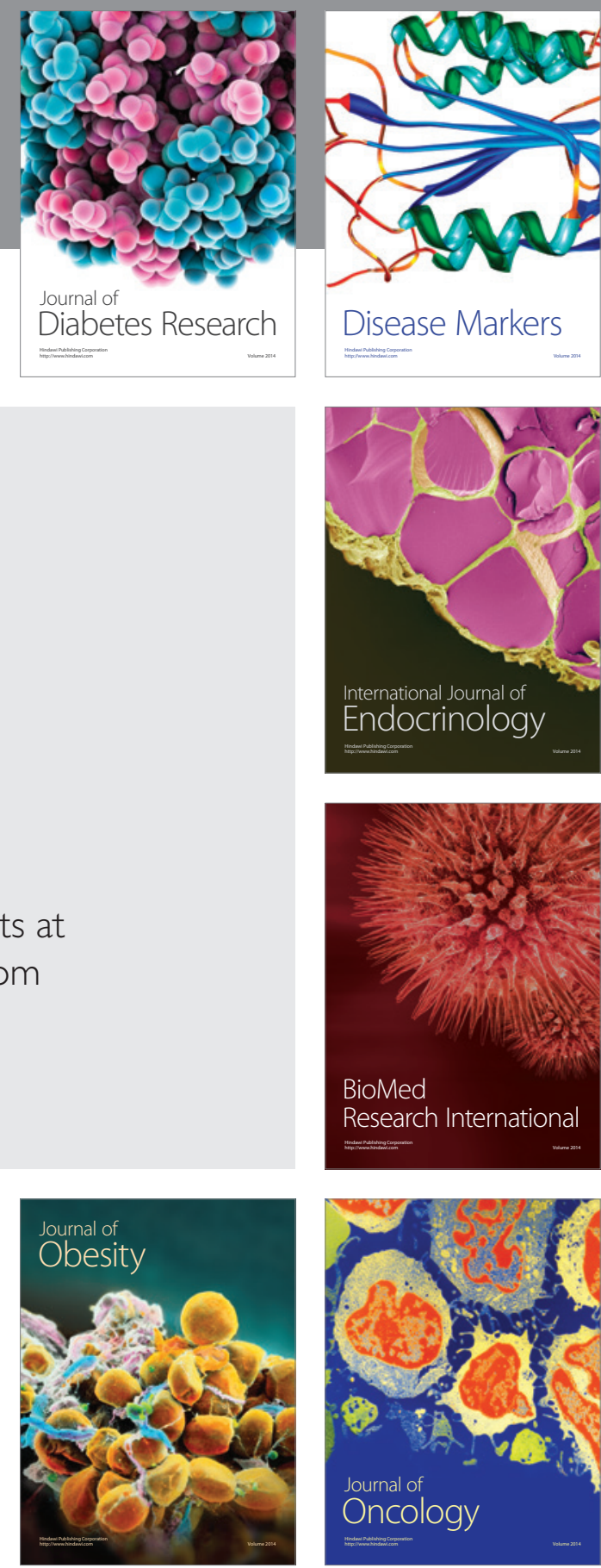

Disease Markers
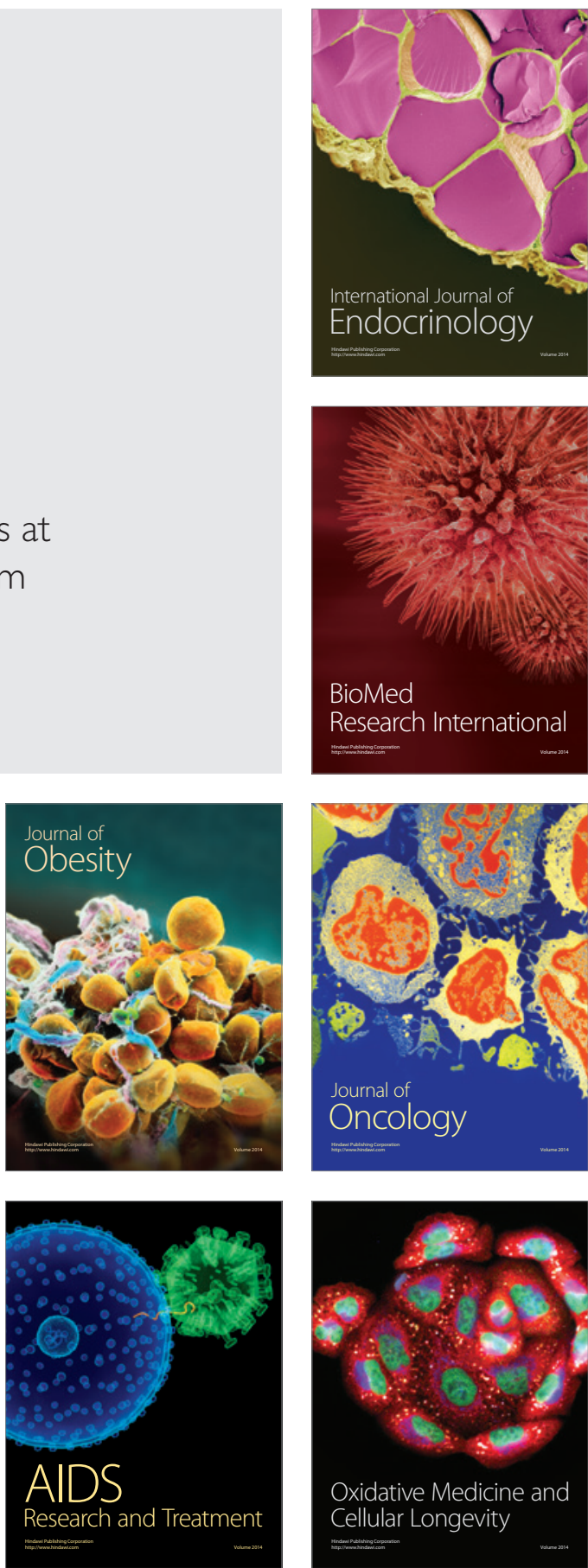